\title{
Variations du nombre de grains chez différents génotypes de maïs
}

\author{
Maurice DERIEUX, Raymond BONHOMME*, Jean-Benoît DUBURCQ, Françoise RUGET* \\ I.N.R.A., Laboratoire de Génétique et d'Amélioration des Plantes \\ (*) Laboratoire de Bioclimatologie, Estrées-Mons-en-Chaussée, +80200 Péronne
}

RÉSUMÉ

\begin{abstract}
Le nombre de grains par épi a été étudié chez 8 hybrides de mais pendant 3 ans, avec des semis échelonnés et, à chaque semis, présence ou non de mulch plastique (au total 22 conditions différentes).

Pour un génotype donné, le nombre de rangs est très peu influencé par le milieu et ne varie pas entre la floraison et la maturité.

Le nombre de grains par rang n'est pas aussi étroitement lié à la précocité que le nombre d'ovules.

Pour un génotype donné, la variation du nombre de grains par rang est reliée essentiellement au nombre d'ovules présents à la floraison pour F7 × F2 et W629A × F2 (types corné et corné-denté) ou au poids sec de la plante à la.floraison pour W64A $\times$ F546 et WH $\times$ WJ (type denté). La température durant les différentes phases de développement n'a pas d'influence sauf pour W64A $\times$ F546.
\end{abstract}

Mots clés additionnels : Croissance, développement, température.

Variation in kernel number in various maize hybrids.

The grain yield of a maize crop is mostly determined by number of kernels, consequently by kernel number per ear. The kernel number per ear was studied on 8 maize hybrids in 22 different environmental conditions ( 3 years, many dates of sowing, use of plastic mulch). Row number was not modified by environmental conditions and did not vary from silking to maturity. Kernel number per row was less related to earliness than ovule number. The genotype mean varied from 300 for $F 7 \times F 2$ to 480 for W64A $\times$ F546. The environmental effect was smaller than the genotype effect but was significant. Among genotypes, variation in kernel number was essentially explained by ovule number at silking for F7 $\times F 2$ and W629 $\times \mathrm{F} 2$ (flint and flint-dent types) and by dry matter weight of the plant at silking for W64A $\times$ F546 and WH $\times$ WJ (dent types). Temperature during different development stages did not affect grain number per row (except for W64A $\times$ F546).

Additional key words : Growth, development, temperature.

\section{INTRODUCTION}

Les différences de production de matière sèche totale entre génotypes de maïs et pour des semis faits en différentes conditions peuvent être reliées à l'énergie solaire utile à la photosynthèse interceptée par le couvert végétal sur les cycles de végétation considérés (BONHOMME et al., 1982). Mais les productions en grains semblent plus difficiles à mettre en relation avec les phénomènes de photosynthèse, et donc avec la production de matière sèche de la culture, comme le montrent les nombreuses discussions sur les relations « source-puits » (TOLLENAAR, 1977). Cependant, en conditions hydriques peu limitantes, il apparaît que les différences de production en grain sont dues essen- tiellement à la variation du nombre de grains par plante (BOIFFIN et al., 1976 ; DERIEUX, 1978).

La variation du nombre de grains observée a 2 origines : le génotype et le milieu. Nous proposons donc dans ce travail d'étudier les variations du nombre de grains chez différents génotypes, semés en zone septentrionale, à différentes dates et avec, dans certains cas, un mulch plastique destiné à accélérer le développement de la culture. La variation observée dans les nombres de grains sera mise en relation avec les conditions de milieu (température essentiellement) mais aussi avec les caractéristiques de la plante à floraison (nombre d'ovules, poids sec, nombre de feuilles) ou au cours de son cycle (durée de différents stades). La comparaison de différents génotypes permettra de 
mettre en évidence d'éventuelles différences de comportement dans la détermination du nombre de grains.

\section{MATÉRIEL ET MÉTHODES}

Les essais ont été réalisés à Péronne (latitude $50^{\circ} \mathrm{N}$, longitude $3^{\circ} \mathrm{E}$, altitude $85 \mathrm{~m}$ ) en 1979,1980 et 1981 . Une présentation détaillée des matériels et méthodes de ces essais a déjà été faite par ailleurs (DUBURCQ $e t$ al., 1983), rappelons seulement les caractéristiques des génotypes :

- F7 $\times$ F2 : hybride simple, très précoce, corné

- WH $\times$ WJ : hybride simple, précoce, denté

- W629A $\times$ F2 (BIP) : hybride simple cultivé, précoce, corné-denté

- (F7 $\times$ F2) F230 (HTV216) : hybride 3 voies, cultivé, précoce, corné-denté, grain riche en protéines

- LG 11 : hybride 3 voies, très cultivé, précoce, corné-denté

- F478 × F564 (HS 222) : hybride simple, précocité moyenne, à tendance prolifique

- W64A x F546 (INRA 508) : hybride simple, cultivé, tardif, denté

- P.T. : hybride expérimental comparable à INRA 508 avec une tendance à la prolificité

- B73 $\times$ Mol7 (noté BMo) : hybride simple, très tardif, représentatif des zones de culture du Corn-Belt américain.

Les notations de nombre de grains ont été faites sur l'épi principal, bien après la floraison mais avant maturité, sur 3 répétitions de 10 plantes. Ce nombre

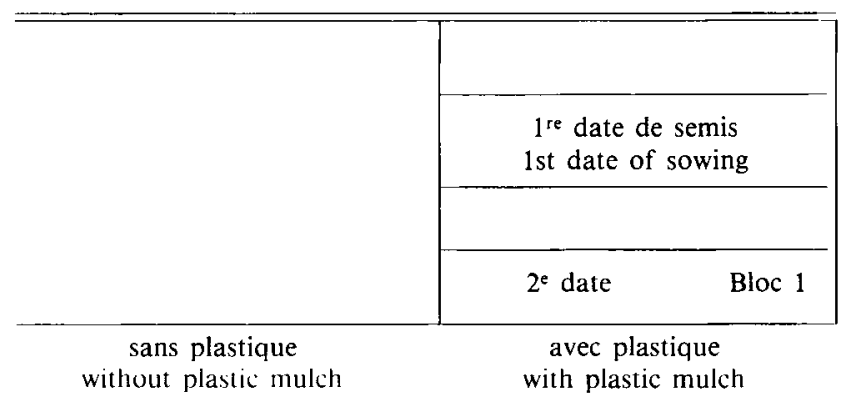

Figure 1

\section{Disposition des essais sur le terrain.} Experimental design.

est le produit du nombre de rangs par le nombre de grains par rang.

Compte-tenu du dispositif utilisé (fig. 1) et en particulier du fait que les traitements mulch plastique sont groupés sur le terrain, la décomposition de la variance par année (tabl. 1) a été faite de la façon suivante (P. VINCOURT, comm. pers.) :

$$
\begin{aligned}
\mathrm{Y}_{\mathrm{ijkl}}= & \mathrm{m}+\mathrm{a}_{\mathrm{i}}+\mathrm{b}_{\mathrm{j}}+\theta_{\mathrm{ij}}+\mathrm{E}_{\mathrm{ij}}+\mathrm{f}_{\mathrm{ijk}}+\mathrm{g}_{\mathrm{l}}+\mathrm{r}_{\mathrm{il}}+ \\
& +\mathrm{t}_{\mathrm{jl}}+\mathrm{U}_{\mathrm{ijl}}+\mathrm{Z}_{\mathrm{ijkl}} \\
\mathrm{i}= & \text { traitement mulch : terrain nu ou couvert de } \\
& \text { plastique } \\
\mathrm{j}= & \text { date de semis } \\
\mathrm{k}= & \text { bloc pour une date de semis avec ou sans } \\
& \text { mulch plastique }=\text { bloc/mulch } \times \text { date } \\
\mathrm{l}= & \text { génotype. }
\end{aligned}
$$

L'interaction mulch $\times$ date $\left(\theta_{i j}\right)$ ne peut être distinguée de l'erreur $\left(\mathrm{E}_{\mathrm{ij}}\right)$ par rapport à laquelle on teste les

\begin{tabular}{|c|c|c|c|c|c|c|c|c|c|c|c|c|c|c|c|c|c|c|c|c|c|c|c|c|}
\hline \multirow{3}{*}{$\begin{array}{l}\text { Année-Year } \\
\text { Date de semis } \\
\text { Mulch plastique }\end{array}$} & \multicolumn{10}{|c|}{1979} & \multicolumn{6}{|c|}{1980} & \multicolumn{6}{|c|}{1981} & \multicolumn{2}{|c|}{$1979-1980-1981$} \\
\hline & \multicolumn{2}{|c|}{$10 / 4$} & \multicolumn{2}{|c|}{$25 / 4$} & \multicolumn{2}{|c|}{$2 / 5$} & \multicolumn{2}{|c|}{$14 / 5$} & \multicolumn{2}{|c|}{$1 / 6$} & \multicolumn{2}{|c|}{$9 / 4$} & \multicolumn{2}{|c|}{$6 / 5$} & \multicolumn{2}{|c|}{$4 / 6$} & \multicolumn{2}{|c|}{$7 / 4$} & \multicolumn{2}{|c|}{$29 / 4$} & \multicolumn{2}{|c|}{$2 / 6$} & \multirow{3}{*}{\multicolumn{2}{|c|}{$\begin{array}{l}\text { Moyenne } \\
\text { écart- } \\
\text { type } \\
\text { coefficient } \\
\text { de variation }\end{array}$}} \\
\hline & $\mathrm{Pl}$ & $\mathrm{Nu}$ & $\mathrm{PI}$ & $\mathrm{N}_{11}$ & $\mathrm{Pl}$ & $\mathrm{N}_{11}$ & $\mathrm{PI}$ & $\mathrm{Nu}$ & $\mathrm{Pl}$ & $\mathrm{Nu}$ & $\mathrm{Pl}$ & $\mathrm{Nu}$ & $\mathrm{Pl}$ & $\mathrm{Nu}$ & $\mathrm{Pl}$ & $\mathrm{Nu}$ & $\mathrm{PI}$ & $\mathrm{Nu}$ & PI & $\mathrm{Nu}$ & $\mathrm{PI}$ & $\mathrm{Nu}$ & & \\
\hline \multicolumn{23}{|l|}{ Génotypes } & & \\
\hline $\mathrm{F} 7 \times \mathrm{F} 2$ & & & 315 & 304 & 285 & 309 & 342 & 312 & 319 & 302 & 276 & 257 & 305 & 242 & 308 & 290 & 325 & 267 & 297 & 323 & 330 & 316 & 301 & $25 \quad 8,2$ \\
\hline$W H \times W J$ & 346 & 322 & 357 & 318 & 341 & 319 & 324 & 282 & 323 & 185 & 267 & 357 & 299 & 353 & 343 & 338 & 381 & 407 & 392 & 371 & 387 & 383 & 336 & $48 \quad 14,3$ \\
\hline BIP & 361 & 366 & 357 & 376 & 358 & 323 & 396 & 304 & 404 & 334 & 276 & 268 & 298 & 276 & 316 & 343 & 346 & 318 & 349 & 335 & 382 & 343 & 338 & $37 \quad 10,9$ \\
\hline INRA 508 & 502 & 467 & 507 & 446 & 505 & 454 & 505 & 499 & & 412 & 470 & 424 & 441 & 371 & 462 & 455 & 567 & 493 & 528 & 503 & 511 & 528 & 478 & $44 \quad 9,1$ \\
\hline HS 222 & 367 & 369 & 365 & 361 & 399 & 377 & 381 & 373 & 378 & 334 & 309 & 331 & 327 & 342 & 393 & 367 & 380 & 399 & 365 & 387 & 434 & 380 & 369 & $37 \quad 7,4$ \\
\hline LG 11 & 383 & 397 & 380 & 396 & 375 & 410 & 409 & 369 & 412 & 379 & 334 & 327 & 341 & 376 & 405 & 398 & & & & & & & 381 & $26 \quad 6,8$ \\
\hline BMo & 377 & 253 & 448 & 253 & 411 & 347 & 443 & 128 & 300 & & 505 & 524 & 517 & 519 & 466 & 465 & & & & & & & 397 & 11528,9 \\
\hline P.T. & & & 345 & 450 & 374 & 423 & 382 & 424 & 414 & 288 & 388 & 374 & 388 & 376 & 441 & 387 & & & & & & & 390 & $40 \quad 10,2$ \\
\hline \multirow[t]{3}{*}{ HTV 216} & 395 & 404 & 380 & 395 & 382 & 363 & 395 & 363 & 430 & 364 & & & & & & & & & & & & & 387 & $20 \quad 5,2$ \\
\hline & & & \multicolumn{6}{|c|}{1979} & \multicolumn{5}{|c|}{1980} & \multicolumn{6}{|c|}{1981} & \multicolumn{5}{|c|}{ Années confondues } \\
\hline & & & \multicolumn{2}{|c|}{$\begin{array}{l}\text { C.M. } \\
\text { (M.S.) }\end{array}$} & $\begin{array}{r}\mathrm{D} \\
\text { (D. }\end{array}$ & $\begin{array}{l}\text { L } \\
\text { F.) }\end{array}$ & \multicolumn{2}{|c|}{ F. } & C.I & & D.L. & & F. & & C.M. & & D.L. & & F. & & C.M. & & D.L. & $F$. \\
\hline Mulch & & & 50 & 403 & & 1 & 7,4 & & & 793 & 1 & & 1,0 & & 5413 & & 1 & & 5,5 & & & & & \\
\hline Date de semis & & & & 785 & & 4 & 0,4 & & 12 & 770 & 2 & & 15,6 & & 1486 & & 2 & & 1,5 & & 3687 & & 21 & $4,2^{* * *}$ \\
\hline Mulch $\times$ date & & & & 784 & & 4 & & & & 819 & 2 & & & & 992 & & 2 & & & & & & & \\
\hline Blocs dans mulch $\times$ date & & & & 123 & & 20 & & & & 641 & 12 & & & & 539 & & 12 & & & & & & & \\
\hline Génotype & & & 74 & 596 & & 8 & 44,5 & $5 * * *$ & 97 & 474 & 7 & & $99,6 * * *$ & & 114371 & & 4 & & $6,3^{* * *}$ & & 8930 & & 4 & $113,9 * * *$ \\
\hline Génotype $\times$ mulch & & & 12 & 495 & & 8 & 7,4 & $4 * * *$ & & 555 & 7 & & $3,6^{* *}$ & & 865 & & 4 & & 1,3 & & & & & \\
\hline Génotype $\times$ date & & & & 053 & & 32 & 1,8 & & & 962 & 14 & & $3,0^{* * *}$ & & 819 & & 8 & & 1,2 & & & & & \\
\hline Génotype $\times$ mulch $\times$ date & & & & 440 & & 32 & & $y^{* * *}$ & & 272 & 14 & & 1,3 & & 2020 & & 8 & & $2,9 * *$ & & & & & \\
\hline Erreur & & & & 677 & 16 & 60 & & & & 979 & 84 & & & & 688 & & 18 & & & & 868 & & 84 & \\
\hline
\end{tabular}
effets sol et date. Cependant en raisonnant sur l'importance du carré moyen mulch $\times$ date vis-à-vis des carrés moyens résiduels et bloc/mulch $\times$ date, on

TABLEAU 1

Variations du nombre de grains chez différents génotypes pour différentes années et conditions de semis : avec $(P /)$ ou sans $(N u)$ mulch plastique.

Variations in kernel number for various genotypes, different years and sowing conditions: with (Pl) or without (Nu) plastic mulch. 
peut penser que les termes $\theta_{i j}$ (interaction «vraie » sol $\times$ date) ne sont pas nuls.

L'analyse de variance « années confondues » considère les 22 conditions de croissance différentes (année, date, mulch) comme des blocs.

Dans l'analyse de variance on a pris pour les données manquantes (semis du 10-4-1979) les résultats correspondant aux conditions de milieu les plus proches pour le génotype donné.

Les astérisques : ${ }^{*}, * *, * * *$, indiquent une différence ou une corrélation significative au seuil 0,$05 ; 0,01$; 0,001 respectivement.

\section{RÉSULTATS ET DISCUSSION}

\section{A. Variations du nombre de grains}

Les résultats des divers semis pour les différents génotypes sont donnés dans le tableau 1. La variation due au milieu est comparable pour tous les génotypes (coefficient de variation de 7 à 14 p. 100), à l'exception de B73 $\times$ Mo17 vraiment trop tardif pour la zone de culture. Il est à noter que cette variation dans les nombres de grains est voisine de celle des nombres d'ovules à floraison (c.v. : 8,1 à 10,3 p. 100) (BONHOMME et al., 1983). Les différences génotypiques sont importantes (de 300 pour F7 $\times$ F2 à 480 pour W64A $\times$ F546) mais beaucoup moins reliées à la précocité $\left(r=0,63^{*}\right)$ que le nombre d'ovules.

L'analyse de variance met toujours nettement en évidence l'effet génotypique, mais beaucoup moins l'influence du milieu : date de semis, année, présence de mulch. Il peut être intéressant pour faciliter la compréhension de la détermination du nombre de grains d'étudier séparément le nombre de rangs et le nombre de grains par rang. RuGET \& DUBURCQ (1983) ont montré que le nombre de rang d'ovules était fixé très tôt après le début de différenciation de l'épi. Par contre, le nombre de grains par rang n'est fixé qu'après la floraison.

\section{B. Variations du nombre de rangs de l'épi}

Les résultats sont donnés dans le tableau 2. La variation due aux conditions de milieu est très faible alors que les différences génotypiques sont nettes. Ce même tableau montre que le nombre de rangs de l'épi n'est pas significativement différent du nombre de rangs d'ovules à floraison.

\section{Variations du nombre de grains par rang}

Si l'on considère l'ensemble des données du tableau 1 sans distinguer les génotypes, le nombre de grains par rang, comme le nombre total de grains, varie essentiellement en fonction de la précocité et des caractéristiques liées à celle-ci : nombre de feuilles, durée levéefloraison, ...

Par contre, si l'on fait les corrélations environnementales hybride par hybride entre le nombre de grains, d'une part, et le nombre total de feuilles, les durées de phase, la température aux différentes périodes, le poids sec à la floraison ou le nombre d'ovules

TABLEAU 2

Variations du nombre de rangs de l'épi de divers génotypes cultivés en différentes conditions. Row number variations for various genotypes grown in different environmental conditions.

\begin{tabular}{|c|c|c|c|c|c|c|c|c|}
\hline Génotypes & $\mathrm{F} 7 \times \mathrm{F} 2$ & $\mathrm{WH} \times \mathrm{WJ}$ & BIP & INRA 508 & HS 222 & LG 11 & BMo & P.T. \\
\hline Moyenne & 11,6 & 11,8 & 13,8 & 14,9 & 12,8 & 12,8 & 14,6 & 12,9 \\
\hline Ecart-type (1) & 0,5 & 0,5 & 0,4 & 0,4 & 0,3 & 0,3 & 0,5 & 0,6 \\
\hline Coefficient de variation $(\%)$ & 4,2 & 3,8 & 2,3 & 2,9 & 2,4 & 2,6 & 3,8 & 4,5 \\
\hline
\end{tabular}

Analyse de variance pour 3 années sur 5 génotypes.

Analysis of variance for 3 years and 5 genotypes

\begin{tabular}{crcc}
\hline \hline Facteur & C.M. & D.L. & F \\
& (M.S.) & (D.F.) & \\
\hline Génotype & 44,0 & 4 & $238,9^{* * *}$ \\
Milieu & 0,3 & 21 & 1,4 \\
Erreur & 0,2 & 84 & \\
\hline
\end{tabular}

Rapports nombre de rangs de l'épi/nombre de rangs d'ovules.

Kernel row number/ovule row number.

\begin{tabular}{lrrrrrrrrr}
\hline \multicolumn{1}{c}{ Génotypes } & F7 $\times$ F2 & WH $\times$ WJ & BIP & INRA 508 & HS 222 & LG 11 & BMo & P.T. \\
\hline Moyenne (\%) & 101,6 & 99,3 & 100,2 & 100,6 & 100,7 & 99,8 \\
Ecart-type S.D. (1) & 5,9 & 3,5 & 2,8 & 3,6 & 3,9 & 3,2 & 3,8 & 6,3 & 6,6 \\
\hline
\end{tabular}

(1) Ecart-type des moyennes pour chaque condition de milieu. 
TABLEAU 3

Coefficients de corrélation entre le nombre de grains par rang et différentes variables, chez divers génotypes. Correlation coefficients between kernel number per row and different variables for various genotypes.

\begin{tabular}{|c|c|c|c|c|c|}
\hline \multirow[b]{2}{*}{ (1) } & $\mathrm{F} 7 \times \mathrm{F} 2$ & $\mathbf{W H} \times \mathbf{W J}$ & $\mathrm{W629A} \times \mathrm{F} 2$ & W64A $\times$ F546 & Nbre de données \\
\hline & & & & & \\
\hline $\begin{array}{l}\text { Température sol levée-différenciation femelle } \\
\text { Mean soil temperature from emergence to female } \\
\text { differentiation }\end{array}$ & $-0,22$ & 0,04 & $-0,12$ & $-0,50$ & 10 \\
\hline $\begin{array}{l}\text { Température air différenciation femelle-floraison } \\
\text { Mean air temperature from female differentiation }\end{array}$ & & & & & \\
\hline $\begin{array}{l}\text { Température air de la décade précédant la floraison } \\
\text { Mean air temperature for ten days before silking }\end{array}$ & $-0,07$ & 0,00 & $-0,17$ & 0,18 & 20 \\
\hline $\begin{array}{l}\text { Température air de la décade suivant la floraison } \\
\text { Mean air temperature for ten days after silking }\end{array}$ & $-0,29$ & $-0,17$ & $-0,38$ & $0,58 * *$ & 20 \\
\hline $\begin{array}{l}\text { Durée levée-floraison (2) } \\
\text { Time from emergence to silking }\end{array}$ & $-0,25$ & 0,04 & -0.25 & 0,17 & 20 \\
\hline Durée différenciation femelle-floraison & & & & & \\
\hline $\begin{array}{l}\text { Nombre de feuilles total } \\
\text { Total leaf number }\end{array}$ & $0,60^{*}$ & $0,64 * *$ & $0,51^{*}$ & 0,14 & 14 \\
\hline $\begin{array}{l}\text { Poids sec à floraison } \\
\text { Dry matter weight at silking time }\end{array}$ & 0,36 & $0,53^{*}$ & $0,47^{*}$ & $0,62^{* *}$ & 20 \\
\hline $\begin{array}{l}\text { Nombre d'ovules à floraison } \\
\text { Ovule number at silking time }\end{array}$ & $0,77 * *$ & 0,25 & $0,61 * *$ & $0,48^{*}$ & 20 \\
\hline
\end{tabular}

(1) Température moyenne.

(2) En jours.

à la floraison, d'autre part (tabl. 3), on constate que les mêmes corrélations n'existent pas chez tous les hybrides. Ces corrélations n'ont été faites que pour les 4 hybrides pour lesquels nous disposions de l'ensemble des observations.

(1)

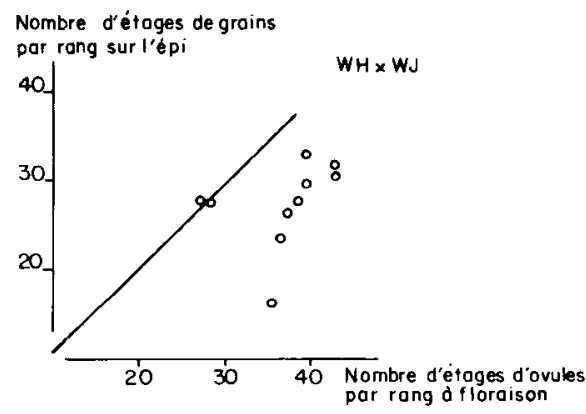

(2)

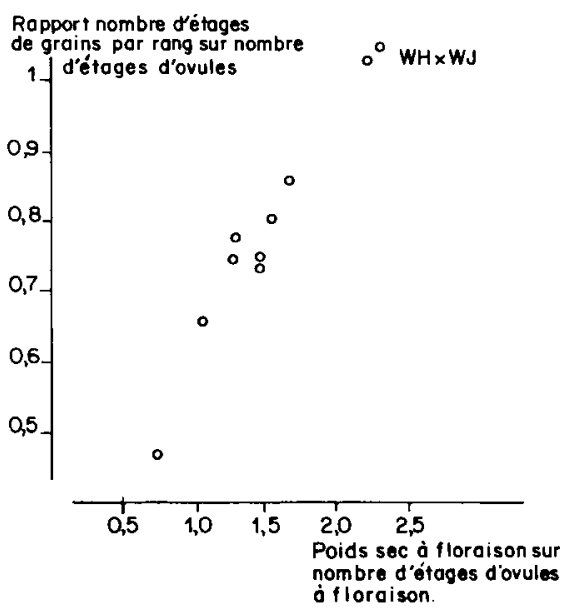

Figure 2

Différence de comportement entre $F 7 \times F 2$ et $W H \times W J$ pour le nombre de grains en fonction du nombre d'ovules par rang (l) et pour le rapport nombre de grains/nombre d'ovules par rang en fonction du poids à la floraison/nombre d'ovules (2).
Selon les génotypes, les meilleures corrélations sont trouvées avec le nombre d'ovules à la floraison $(\mathrm{F} 7 \times \mathrm{F} 2, \mathrm{~W} 629 \mathrm{~A} \times \mathrm{F} 2)$, avec le poids sec à la floraison $(\mathrm{W} 64 \mathrm{~A} \times \mathrm{F} 546, \mathrm{WH} \times \mathrm{WJ}, \mathrm{W} 629 \times \mathrm{F} 2)$, avec le nombre total de feuilles $(\mathrm{WH} \times \mathrm{WJ}, \mathrm{F} 7 \times \mathrm{F} 2)$ et
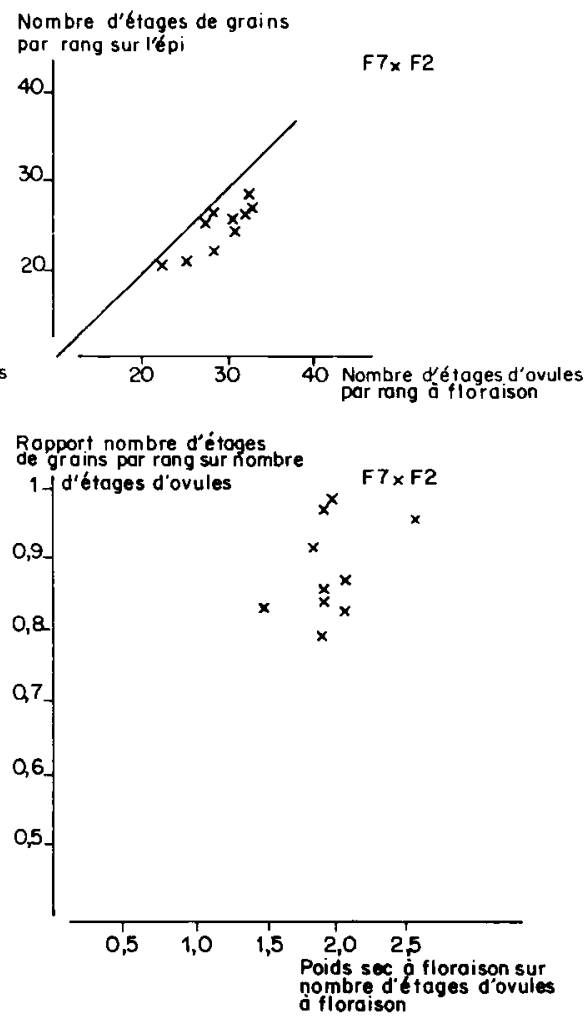

Behaviour differences between $F 7 \times F 2$ and $W H \times W J$, concerning kernel number related to ovules number per row (l) and the ratio kernel number/ovule number per row related to dry matter weight at silking/ovule number (2). 
avec les durées de phases (ou températures avant et après floraison) : WH $\times$ WJ, W64A $\times$ F546.

Une tendance semble se dégager : le facteur le plus important pour la détermination du nombre de grains est le nombre d'ovules présents à la floraison pour F7 $\times$ F2 et W629 $\times$ F2 (type corné et corné-denté), le poids sec à la floraison pour W64A $\times$ F546 et WH $\times$ WJ (type denté). A noter que, pour ces 2 derniers génotypes, le rapport (nombre de grains par rang)/(nombre d'ovules par rang) est très bien relié au rapport (poids sec à floraison)/(nombre d'ovules par rang) $: r=0,63^{* *}$ et $0,64^{* *}$. La figure 2 illustre, en ne considérant que les semis en terrain nu, le comportement très différent de 2 génotypes pour la détermination du nombre de grains.

Bien qu'il ne soit pas possible de faire des comparaisons strictes, la liaison qui existe dans certaines de nos observations entre nombre de grains et matière sèche à la floraison est à relier aux travaux de HAWKINS \& COOPER (1981) sur les relations entre nombre de grains et accroissements de poids avant floraison. La disponibilité en assimilats avant floraison dans la détermination du nombre de grains est aussi mis en évidence dans les relations entre pourcentage de fécondation et teneur en sucres de la tige (WILLIAMS et al., 1968 ; IWATA, 1975).

L'originalité de notre travail est de mettre en évidence des différences de comportement très nettes entre génotypes. Ce qui a des conséquences importantes sur la détermination des objectifs de sélection.
Les corrélations observées sont rarement très fortes, ce travail doit donc être poursuivi pour préciser, selon les génotypes, la transformation précise de l'ovule en grain et les raisons qui peuvent mettre fin à cette évolution. La fécondation peut ne pas avoir lieu pour diverses raisons : ovules non fonctionnels, non sortie des soies, soies non fonctionnelles, durée trop grande entre fécondation des grains de la base et sortie des dernières soies (problèmes de dominance hormonale). Les grains peuvent avorter après fécondation pour des raisons de compétition purement trophique ou liées à des relations hormonales (gradient sur l'épi). Ces relations entre grains du même épi peuvent être comparées aux relations entre épis d'une même plante (GALLAIS et al., 1982).

Ces observations donnent des informations intéressantes sur les relations source-puits. Il existe une source et un puits potentiels déterminés génétiquement. Les conditions de milieu permettent toujours la réalisation du « nombre de rangées d'ovules » potentiel. Chez un génotype comme F7 $\times$ F2, la source potentielle serait limitée génétiquement, le milieu permettrait toujours plus ou moins sa réalisation, le nombre d'ovules (taille du " puits ») s'ajusterait alors à l'efficacité de la source foliaire réalisée. Chez WH $\times$ WJ ou W64A $\times$ F546 (type denté), la "source foliaire " potentielle est plus importante et plus difficile à réaliser, et l'ajustement définitif de la taille du puits ne se ferait qu'à partir de la transformation des ovules en grains.

\section{RÉFÉRENCES BIBLIOGRAPHIQUES}

Boiffin J., Sebillotle M., Couvreur F., 1976. Incidence de la simplification du travail du sol sur l'élaboration des rendements du blé et du maïs. Colloque ITCF « Simplification du travail du sol en conditions céréalières », 239-280.

Bonhomme R., Ruget F., Derieux M., Vincourt P., 1982. Relations entre production de matière sèche aérienne et énergie interceptée chez différents génotypes de maïs. C.R. Acad. Sci. Paris, série III, 294, 393-398.

Bonhomme K., Derieux M., Duburcq J.-B., Ruget F., 1984. Variations in ovule number at silking in various corn genotypes. Maydica, XXIX, 101-107.

Derieux M., 1978. Etude de quelques facteurs d'adaptation du maïs aux conditions climatiques du Nord de la France. Ann. Amélior. Plantes, 28 (5), 529-566.

Duburcq J.-B., Bonhomme R., Derieux M., 1983. Durée des phases végétative et reproductrice chez le maïs. Influence du génotype et du milieu. Agronomie, 3 (10), 941-946.
Gallais A., Kellerhals M., Philippe F., 1982. Etude des interactions entre épis chez le maïs. Agronomie, 2 (10), 995-1004.

Hawkins R. C., Cooper P. J. M., 1981. Growth, development and grain yield of maize. Expl. Agric., 17, 203-207.

Iwata F., 1975. Ear barrenness of corn as affected by plant population. Japan Agric. Res. Quartely, 9, ${ }^{\circ} 1$.

Ruget F., Duburcq J.-B., 1983. Développement reproducteur des bourgeons axillaires chez le maïs : stade de différenciation, nombre de fleurs. Agronomie, 3 (8), 797-807.

Tollenaar M., 1977. Sink-source relationships during reproductive development in maize. A review. Maydica, XX11, 49-75.

Williams W. A., Loomis R. S., Duncan W. G., Dovrat A., Nunez F., 1968. Canopy architecture at various population densities and the growth and grain yields of corn. Crop Sci., 8, 303-308. 